\title{
Experimental Investigation on Acousto-ultrosonic Sensing Using Polarization-maintaining Fiber Bragg Gratings
}

\author{
Gang Wang ${ }^{1}$ \\ University of Alabama in Huntsville, Huntsville, AL, 35899 \\ Curtis E. Banks ${ }^{2}$ \\ NASA Marshall Space Flight Test Center, Huntsville, AL, 35811
}

\begin{abstract}
This report discusses the guided Lamb wave sensing using polarization-maintaining (PM) fiber Bragg grating (PM-FBG) sensor. The goal is to apply the PM-FBG sensor system to composite structural health monitoring (SHM) applications in order to realize directivity and multi-axis strain sensing capabilities while using reduced number of sensors. Comprehensive experiments were conducted to evaluate the performance of the PM-FBG sensor in a composite panel structure under different actuation frequencies and locations. Three MacroFiber-Composite (MFC) piezoelectric actuators were used to generate guided Lamb waves and they are oriented at 0,45 , and 90 degrees with respect to PM-FBG axial direction, respectively. The actuation frequency was varied from $20 \mathrm{kHz}$ to $200 \mathrm{kHz}$. It is shown that the PM-FBG sensor system is able to detect high-speed ultrasound waves and capture the characteristics under different actuation conditions. Both longitudinal and lateral strain components in the order of nano-strain were determined based on the reflective intensity measurement data from fast and slow axis of the PM fiber. It must be emphasized that this is the first attempt to investigate acouto-ultrosonic sensing using PM-FBG sensor. This could lead to a new sensing approach in the SHM applications.
\end{abstract}

\section{Nomenclature}

$n_{0}=$ Nominal index of refraction of the fiber core $\left(\mathrm{n}_{0}=1.46\right)$

$p_{11}, p_{12}=$ Strain-optic coefficients $\left(\mathrm{p}_{11}=1.21 ; \mathrm{p}_{12}=0.27\right)$

$v \quad=$ Poisson's ratio of fiber

$\varepsilon_{x x} \quad=$ Normal strain along fiber axial direction

$\varepsilon_{y y} \quad=\quad$ Normal strain along slow axis (i.e., lateral)

$\varepsilon_{z z}=$ Normal strain along fast axis (i.e., transverse)

$\lambda_{\mathrm{f}}=$ Initial unstrained wavelength of fast axis $\left(\lambda_{\mathrm{f}}=1549.563 \mathrm{~nm}\right)$

$\lambda_{\mathrm{s}}=$ Initial unstrained wavelength of slow axis $\left(\lambda_{\mathrm{s}}=1550.120 \mathrm{~nm}\right)$

$\lambda_{\mathrm{B}} \quad=$ Bragg wavelength

$\Delta \quad=$ Grating pitch

$\Delta \lambda_{\mathrm{f}} \quad=$ Wavelength shift along fast axis when strained

$\Delta \lambda_{\mathrm{s}} \quad=$ Wavelength shift along slow axis when strained

\section{Introduction}

$\mathrm{C}$ omposites structures are increasingly being used in the construction of aerospace, mechanical, civil, marine, automotive, and other high performance structures due to their high specific stiffness and strength, excellent fatigue resistance, longer durability as compared to their metallic counterparts, and due to their ability to be easily tailored for

\footnotetext{
${ }^{1}$ Ph.D., Assistant Professor, Department of Mechanical and Aerospace Engineering, gang.wang@uah.edu
}

${ }^{2}$ Ph.D., MSFC-E35, BLD 4487/B102 
specific applications. One example is the composite over-wrapped pressure vessels (COPVs) ${ }^{1}$, which are currently used at NASA to contain high-pressure fluids in propulsion, science experiments and life support applications, as shown in Figure 1. These COPVs have a significant weight advantage over all-metal vessels; but, as compared to allmetal vessels, COPVs require unique design, manufacturing, and test requirements. COPVs are in general subjected to significant multi-axial, dynamic state of stress and can be of significant risk to structural damages. Damage analysis, diagnostics, and life predictions are key factors in the structural health monitoring (SHM) of COPVs.

The Acousto-ultrasonic (AU) approach has been used to identify damages in engineering structures ${ }^{2-7}$. Worlton proposed an AU type approach for damage detection in structures ${ }^{3}$. Rose has presented a literature review of the most salient work with regard to AU research ${ }^{4}$. Dalton conducted studies of Lamb wave propagation through aircraft structures and noted that long range inspection is possible ${ }^{5}$. Cawley and Alleyne discussed the different Lamb modes present

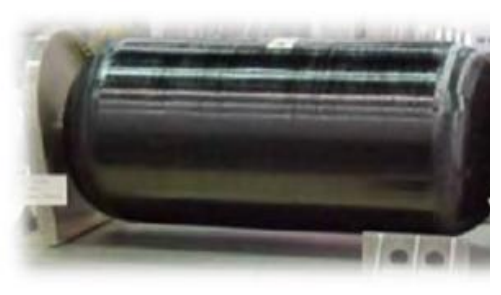

a). Nitrogen Tank

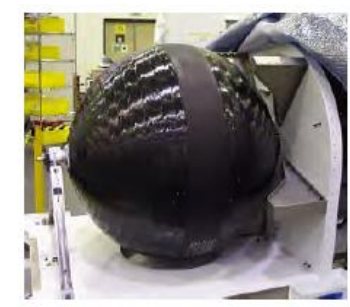

b). Oxygen Tank Figure 1: Carbon-epoxy COPVs in thin plates and their applicability toward damage detection. It is mentioned that dispersion, the change in the shape of the waveform as the wave propagates through the structure, is an important factor in choosing a wave mode for AU damage detection ${ }^{6}$. Wilcox et al. extends Cawley's thoughts about factors critical for AU inspection of structures ${ }^{7}$. Recent advancements in this area including applications of guided ultrasonic Lamb waves for identification of damage in composite structures are discussed in Ref. 8.

Effective and efficient sensors play a major role in order to achieve the in-situ AU damage detection for composite structures. Piezoelectric based sensors have proven to be suitable to this application because of superior electro-mechanical coupling property and high frequency sensing capability. Lead Zirconate Titanate (PZT) is a typical piezoelectric transducer that is extensively used in guided Lamb wave studies ${ }^{9-10}$. PZT transducers are advantageous as they have wide frequency responses, excellent mechanical strength, low power consumption and acoustic impedance. They are light weight and low cost as well. As the counterparts of piezoelectric sensors, fiber Bragg grating (FBG) sensors also offer many benefits including small size, light weight, immunity to electromagnetic-interference, amenability to multiplexing, and high sensitivity ${ }^{11-13}$. The FBG strain sensor consists of a traditional silicon glass fiber upon which a Bragg grating is created. When the Bragg grating is strained, the wavelength reflected by the grating changes linearly ${ }^{14}$. Abroad range of static and dynamic strains can be accurately measured with fiber optic Bragg grating sensors capable of measuring strains as small as $1 \mu \varepsilon^{15}$. A true distributed sensor with multiple (4 to 16) Bragg gratings can be serially multiplexed on one single cable. The capability of Lamb wave sensing using FBGs was demonstrated by testing a Perspex plate under PZT actuation as discussed by Betz et $a l .{ }^{16}$.

The multiplexing feature of FBGs allows us collect strain data at multiple locations simultaneously. However, due to directional dependent properties in composites, multi-axis strain measurements are needed in order to comprehensively evaluate the health of a composite structure. Therefore, a multi-parameter fiber optic strain sensor is required. Two-parameter FBG strain sensors were developed by writing a single fiber Bragg grating onto a polarization-maintaining (PM) optical fiber ${ }^{17-20}$. The birefringence results in a slight change in the index of refraction along two mutually perpendicular directions ${ }^{20}$, termed the polarization axes (i.e., fast and slow axis). By measuring different reflective intensity in both polarization axes, one can determine the corresponding wavelength shifts of Bragg peaks and derive the biaxial strain field. A tri-axial strain can be realized by writing two distinct gratings into one polarization fiber ${ }^{20-21}$. Mawatari and Nelson developed a model to predict longitudinal and two orthogonal transverse strain components from measured wavelength shifts and experimentally validate the model prediction by conducting combined loading test on the fiber ${ }^{21}$.

This study focuses on the application of the PM-FBG sensor to conduct guided Lamb wave sensing in a composite panel structure. The goal is to advance the state-of-the-art of multi-axis sensing in composite SHM applications. We expect to measure in-plane 2D strain fields using a single PM-FBG sensor. To authors' best knowledge, such approach has not been reported in the literature. The PM-FBG sensor performance in terms of frequency bandwidth, directivity, sensitivity, accuracy, is expected to be characterized. Macro Fiber Composite (MFC) piezoelectric actuators were used to excite the composite plate by applying a 5-cycled Hanning windowed sinusoidal signal under various excitation frequencies. Based on the collected reflective intensity measurements in both fast and slow axis, the 2-D strain field can be determined by relating the strain components to the wavelength shifts. 
The report is organized as follows. In section 2, a brief introduction to FBG and PM-FBG is presented. Section 3 will discuss the acousto-ultrasonic experimental setup and procedure to determine the wavelength shifts. Results will be included in Section 4 and conclusion remarks are given in Section 5.

\section{Polarization-maintaining Fiber Bragg Grating}

\section{A. Fiber Bragg Grating}

As shown in Figure 2, a fiber Bragg grating ${ }^{12}$ operates by acting as a wavelength selective filter that reflects a narrow band of light centered on the grating's characteristic wavelength referred to as the Bragg wavelength, $\lambda_{\mathrm{B}}$. The Bragg wavelength is related to the grating pitch, $\Lambda$, and the mean refractive index of the core, $\mathrm{n}_{0}$, by $\lambda_{\mathrm{B}}=2 \Lambda \mathrm{n}_{0}$. Both the fiber refractive index and the grating pitch vary when strain is applied to the FBG and/or the temperature is changed. Wavelength change measurement then provides a basis for strain and temperature sensing.

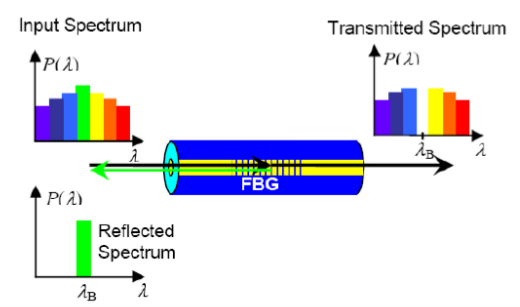

Figure 2: Operational principle of a FBG sensor

\section{B. Polarization-Maintaining FBG}

There are three main types of polarization-maintaining (PM) fiber, i.e., elliptical, bow-tie, and panda type. The polarization axes are setup by applying stress-inducing claddings ${ }^{20}$. Then, the birefringence in the fiber is created with this built-in residual stress introduced during the fiber draw. Figure 3 shows the schematic of the acousto-ultrasonic sensing using a PM-FBG sensor on a plate structure, in which a bow-tie PM fiber is employed. The ultimate goal is to derive the in-plane strain components along axial and lateral directions. First, a calibration is required to relate the wavelength to

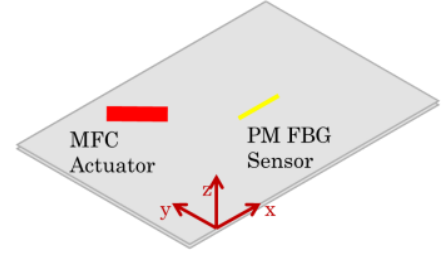

(a). actuator and sensor

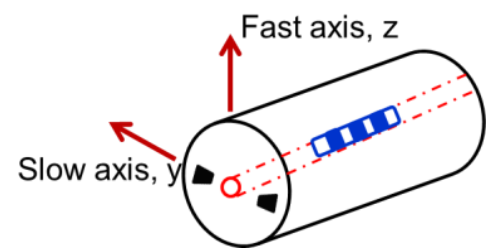

(b) bow-tie PM-FBG

Figure 3: Schematic of Lamb wave sensing using PM-FBG

reflective intensity using an optical spectrum analyzer. Then, we need to collect corresponding reflective intensity responses in both polarization axes when the plate is strained. Finally, the in-plane strain components can be calculated. The strain applied to the grating will result in a shift in the wavelength of two peaks from both fast and slow axis, which is given by

$$
\begin{aligned}
& \frac{\Delta \lambda_{f}}{\lambda_{f}}=\left(1-\frac{n_{0}^{2}}{2} p_{12}\right) \varepsilon_{x x}-\frac{n_{0}^{2}}{2} p_{12} \varepsilon_{y y}-\frac{n_{0}^{2}}{2} p_{11} \varepsilon_{z z} \\
& \frac{\Delta \lambda_{s}}{\lambda_{s}}=\left(1-\frac{n_{0}^{2}}{2} p_{12}\right) \varepsilon_{x x}-\frac{n_{0}^{2}}{2} p_{11} \varepsilon_{y y}-\frac{n_{0}^{2}}{2} p_{12} \varepsilon_{z z}
\end{aligned}
$$

Recall that the out-of-plane stress $\left(\sigma_{\mathrm{zz}}\right)$ is zero. Corresponding out-of-plane strain $\left(\varepsilon_{\mathrm{zz}}\right)$ can be expressed in terms of in-plane strain components as shown below

$$
\varepsilon_{z z}=\frac{-v}{1-v}\left(\varepsilon_{x x}+\varepsilon_{y y}\right)
$$

Note that the optical fiber is assumed to be isotropic. The final expression between in-plane strain components and the shifts in the wavelength is given by

$$
\left[\begin{array}{l}
\varepsilon_{x x} \\
\varepsilon_{y y}
\end{array}\right]=\left[\begin{array}{ll}
k_{11} & k_{12} \\
k_{21} & k_{22}
\end{array}\right]^{-1}\left[\begin{array}{c}
\frac{\Delta \lambda_{f}}{\lambda_{f}} \\
\frac{\Delta \lambda_{s}}{\lambda_{s}}
\end{array}\right]
$$

where

$$
k_{11}=1-p_{12} \frac{n_{0}^{2}}{2}+p_{11} \frac{n_{0}^{2} v}{2(1-v)} \quad k_{12}=-p_{12} \frac{n_{0}^{2}}{2}+p_{11} \frac{n_{0}^{2} v}{2(1-v)} \quad k_{21}=1-p_{12} \frac{n_{0}^{2}}{2}+p_{12} \frac{n_{0}^{2} v}{2(1-v)} \quad k_{12}=-p_{11} \frac{n_{0}^{2}}{2}+p_{12} \frac{n_{0}^{2} v}{2(1-v)}
$$




\section{Experimental Test}

\section{A. Setup}

Figure 4a shows a composite plate with surfaced bonded MFC actuators (M2503-P1 from Smart Material Corp.) and a PM-FBG sensor (TimberCon FBG PM Bowtie, 1550nm). The composite plate is made of IM7 material with 18 cross-ply layup. The plate is $24 \mathrm{in}$. long and $11 \mathrm{in}$. wide. It was hung in the air to create the free edge boundary conditions. Three MFC actuators were oriented at 0,45 , and 90 degrees with respect to the axial direction of the PMFBG sensor in order to investigate the directional sensing capability, which are labeled as MFC 1, MFC 2, and MFC 3. The active size of each MFC is $25 \mathrm{~mm}$ long and $3 \mathrm{~mm}$ wide. Majority strain will be induced along its length direction. Figure $4 \mathrm{~b}$ shows the schematic of the demodulation system to collect the reflective intensity responses from both fast and slow axis. In all tests, the temperature effect on the PM-FBG sensor is neglected because the temperature fluctuation in the laboratory environment is very small.

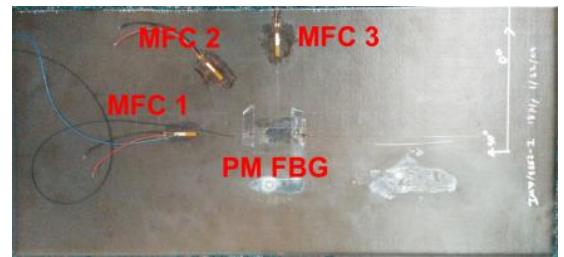

(a).

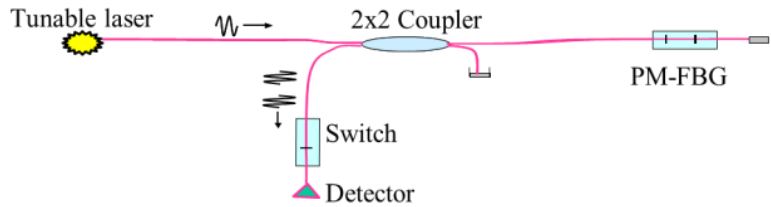

(b)

Figure 4: Experimental setup: (a). composite plate with in-situ actuators and PM-FBG; b). schematic of demodulation system

\section{B. Procedure}

As aforementioned, there are three key steps to derive inplane strain field as outlined below.

Step 1: Determine the grating spectrum

The goal is to obtain the wavelength peaks in polarization axes and calibrate the response curve between the wavelength and reflective intensity. Figure 5 shows the PM-FBG spectrum responses. Clearly, we can identify two peaks which separate the fast axis and slow axis. Ideally, the laser should be set to the wavelength at full width at half-maximum (FWHM) as for the regular FBG sensing ${ }^{16}$. Accordingly, the driving wavelength for the fast axis was set at $1549.563 \mathrm{~nm}$ and the wavelength of $1550.120 \mathrm{~nm}$ was applied to the slow axis.

\section{Step 2: Collect the reflective intensity}

Five-cycled Hanning windowed sine burst was applied to the MFC actuator in order to reduce the side lobes ${ }^{22-23}$. The peak amplitude was 175 volts. Comprehensive tests were performed to collect the reflective intensity responses at different actuating frequencies, ranging from $20 \mathrm{kHz}$ to $200 \mathrm{kHz}$ and repeated the testing for each MFC actuator.

Step 3: Conduct post-processing to calculate strain

Based on the results from step 1 and 2, we can calculate the strain responses using Eq. 1-3.

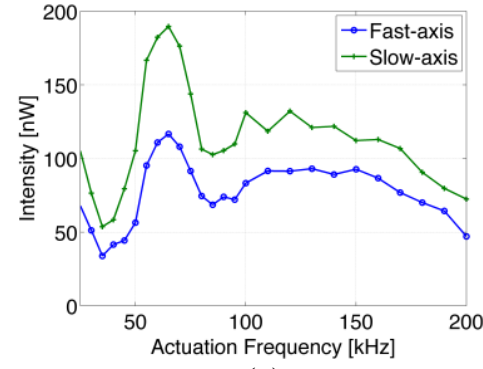

(a)

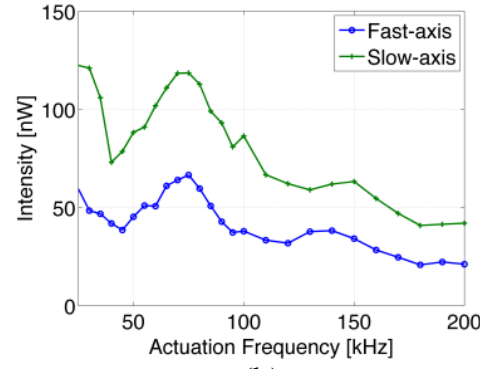

(b)

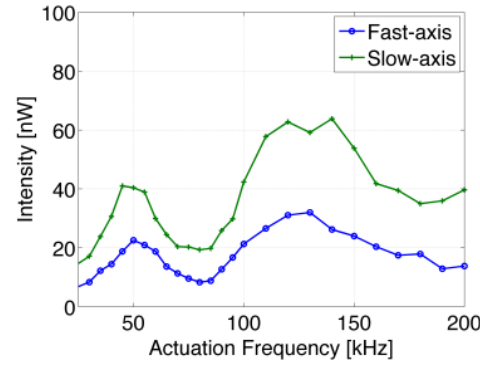

(c)

Figure 6: Reflective intensity responses: (a). 0 degree MFC; (b). 45 degree MFC; and (c) 90 degree MFC 


\section{Results}

Figure 6 shows the frequency responses of the reflective intensity in both fast and slow axis for each actuation case. Clearly, the intensity responses vary with the excitation frequency and reach peak values at certain frequencies, i.e., impedance matching frequencies. As illustrated in Figure 6, the PM-FBG shows directivity because the grating is more sensitive to the induced strain along the fiber axis. The reflective intensity magnitude reduces as the angle of between the MFC actuator and PM-FBG sensor increases from 0 to 90 degrees. A unimorph actuation scheme was employed. Therefore, both antisymmetric modes (i.e., A0, A1, A2, etc.) and symmetric modes (i.e., S0, S1, S2, etc.) were excited ${ }^{2,8,24}$. The wave speed of the first symmetric mode (S0) is much higher than antisymmetric mode (A0). Also, the $\mathrm{S} 0$ wave is less dispersive. In the following sections, we apply these basic understandings of Lamb wave to interpret the measurement data.

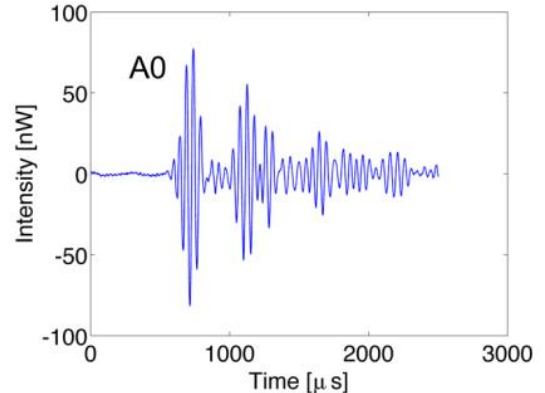

(a)

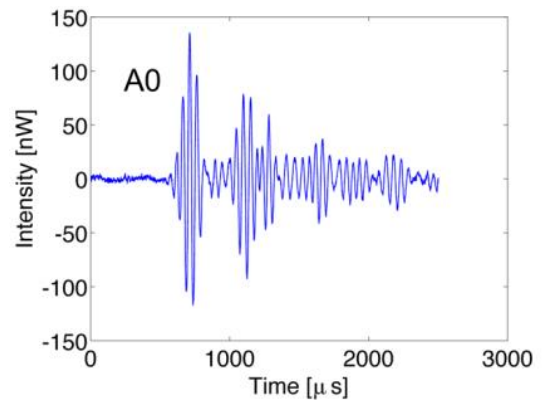

(b)

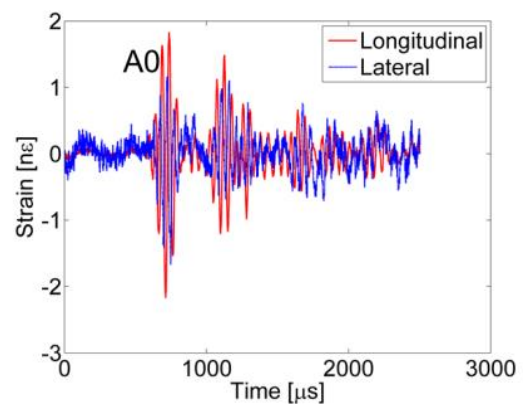

(c)

Figure 7: Reflective intensity and strain responses under $20 \mathrm{kHz}$ actuation using 0 degree MFC: (a). fast axis; (b). slow; and (c) in-plane strain field

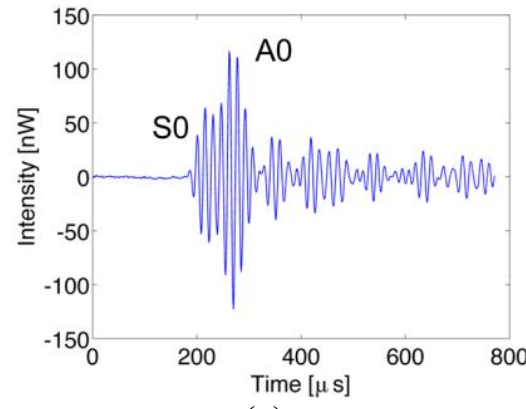

(a)

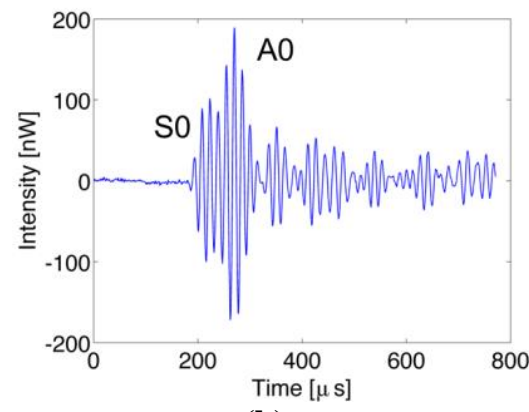

(b)

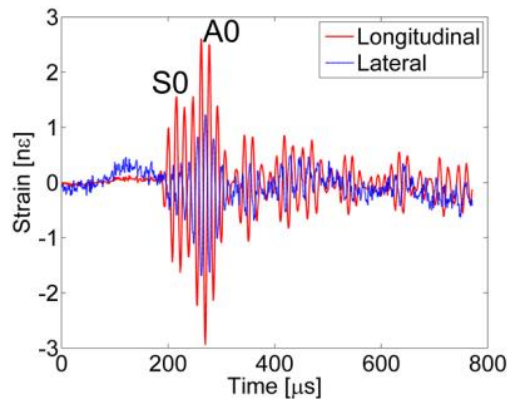

(c)

Figure 8: Reflective intensity and strain responses under $65 \mathrm{kHz}$ actuation using 0 degree MFC: (a). fast axis; (b). slow; and (c) in-plane strain field

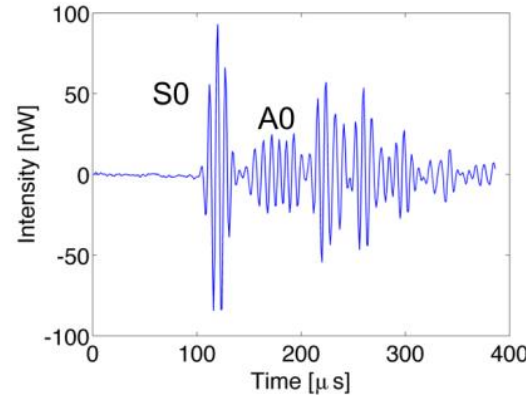

(a)

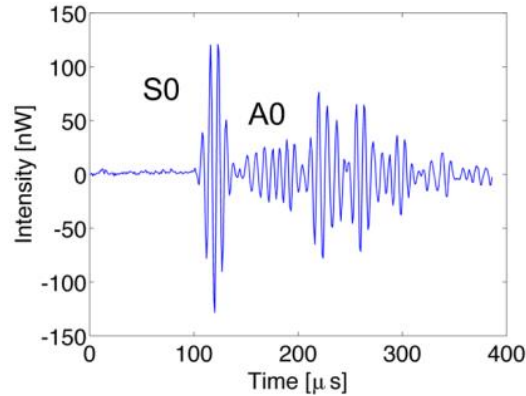

(b)

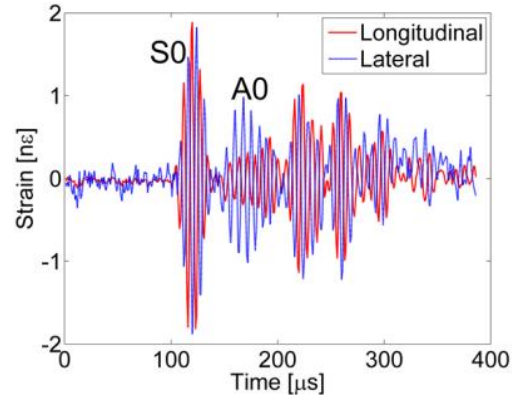

(c)

Figure 9: Reflective intensity and strain responses under $130 \mathrm{kHz}$ actuation using 0 degree MFC: (a). fast axis; (b). slow; and (c) in-plane strain field

Figure 7-9 show reflective intensity and strain time responses of $20 \mathrm{kHz}, 65 \mathrm{kHz}$, and $130 \mathrm{kHz}$, respectively, in which the Lamb wave was generated by MFC 1 (shown in Figure 4a). For the $20 \mathrm{kHz}$ case (Figure 7), the A0 mode was dominant and it decays as it reflects from the edges of the composite plate. The longitudinal strain responses are slightly higher than the lateral responses. As we increase the actuation frequency to $65 \mathrm{kHz}$ (Figure 8), we observe both $\mathrm{A} 0$ and $\mathrm{S} 0$ wave responses. The amplitude of $\mathrm{A} 0$ wave is larger than the amplitude of S0 wave. But, the A0 wave 
is more dispersive as it propagates. There are some interactions between two wave modes. Clearly, both wave modes separate at $130 \mathrm{kHz}$ (Figure 9). Again, the $\mathrm{S} 0$ wave is less dispersive compared to the A0 wave mode. The strain responses under $65 \mathrm{kHz}$ frequency actuation show higher amplitudes compared to the cases of $20 \mathrm{kHz}$ and $130 \mathrm{kHz}$, which is consistent to the reflective intensity frequency responses as shown in Figure 6.

The $70 \mathrm{kHz}$ results actuated by MFC 2 are shown in Figure 10. Similar trends are observed as shown in Figure 8, in which both A0 and S0 wave modes are excited. The $140 \mathrm{kHz}$ results actuated by MFC 3 are shown in Figure 11. The S0 wave is dominant. However, the reflective amplitudes are larger than the instantaneous wave response due to the intensification after the reflection from boundaries. In this case, the MFC actuator is perpendicular to the PM fiber axis. The reflective intensity responses in both fast and slow axis are substantially reduced compared to the cases actuated by MFC 1 and 2. The lateral strain responses are slightly larger than the longitudinal strains as shown in Figure 11c to illustrate the directivity of the PM-FBG sensor.

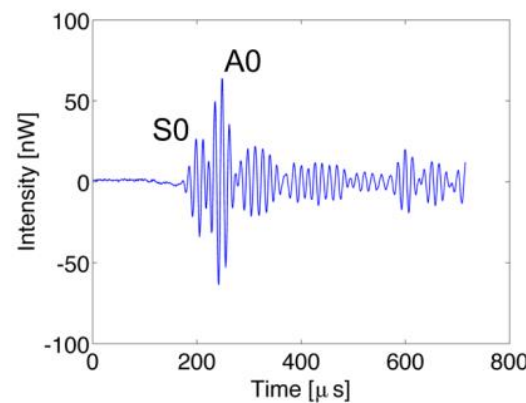

(a)

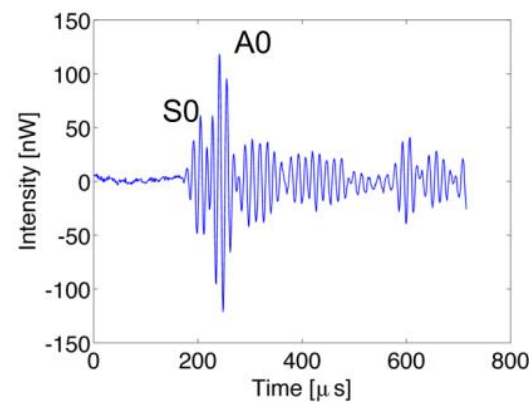

(b)

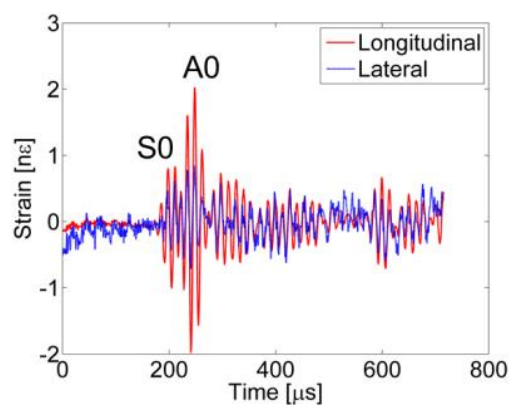

(c)

Figure 10: Reflective intensity and strain responses under $70 \mathrm{kHz}$ actuation using 45 degree MFC: (a). fast axis; (b). slow; and (c) in-plane strain field

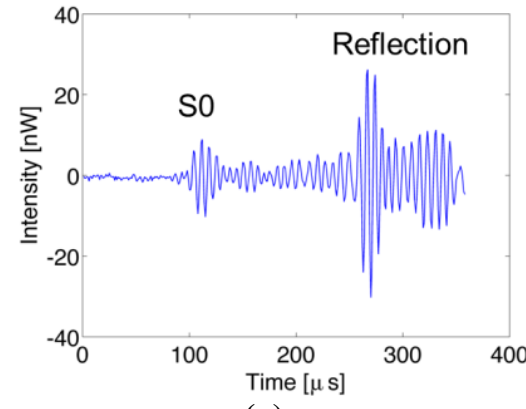

(a)

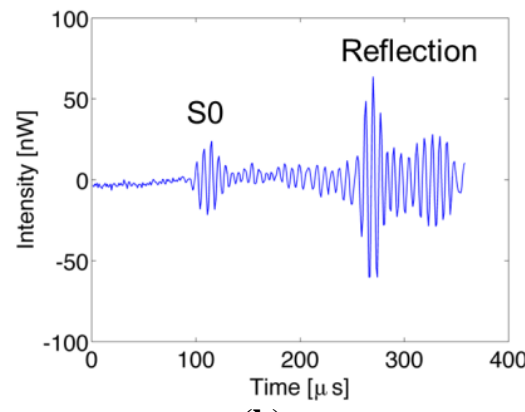

(b)

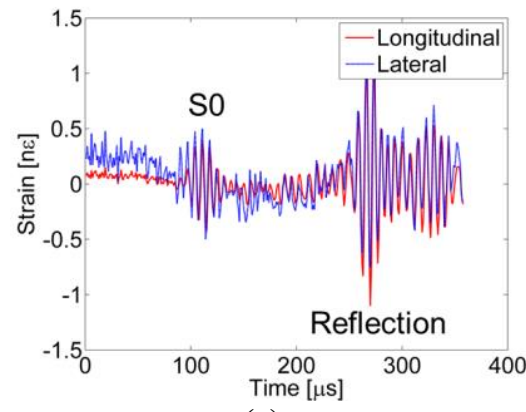

(c)

Figure 11: Reflective intensity and strain responses under $140 \mathrm{kHz}$ actuation using 90 degree MFC: (a). fast axis; (b). slow; and (c) in-plane strain field

\section{Conclusion}

This is the first attempt to demonstrate the acousto-ultrasonic sensing using a PM-FBG sensor in a composite plate. The PM-FBG senor is able to capture both Lamb wave propagation characteristics and derive associated 2-D strain field based on measured reflective intensity responses in both fast and slow axis. The PM-FBG sensor can collect reflective intensity data up to $200 \mathrm{kHz}$ using the proposed demodulation system. The detectable strain is in the range of nano-strain. It is clearly demonstrated that one PM-FBG sensor is able to characterize two-axis strain responses. Therefore, at least 50\% reduction on the sensor number can be achieved, which is substantial in the SHM applications.

\section{Acknowledgments}

Authors would like to thank Claude Chris Conn and Rush Elkins for their assistance to bond actuators and PMFBG. 
NASA - Final Report

\section{References}

${ }^{1}$ Smart COPV" Composite Overwrapped Pressure Vessels (COPVs) Integrated with Structural Health Monitoring (SHM) Systems That Target Space Exploration and ISS Needs, NASA Technical Report, JSC-CN-26776, August 13, 2012.

2 Viktorov, I. A., Rayleigh and Lamb Waves-Physical Theory and Applications, Plenum Press, New York, NY, 1967.

${ }^{3}$ Worlton, D. C., "Ultrasonic testing with Lamb waves," Nondestructive Testing, Vol.15, pp. 218-222, 1957.

${ }^{4}$ Rose, J. L., "A baseline and vision of ultrasonic guided wave inspection potential," Journal of Pressure Vessel Technology, Vol. 124, pp. 273-282, 2002.

${ }^{5}$ Dalton, R. P., Cawley, P., and Lowe, M. J. S. "The potential of guided waves for monitoring large areas of metallic aircraft fuselage structure," Journal of Nondestructive Evaluation, 20(1), pp. 29-46, 2001.

${ }^{6}$ Cawley, P. and Alleyne, D., "The use of Lamb waves for the long range inspection of large structures," Ultrasonics, Vol. 34, pp. 287-290, 1996.

${ }^{7}$ Wilcox, P. D., Lowe, M. J. S., and Cawley, P., "Mode and transducer selection for long range Lamb wave inspection," Journal of Intelligent Material Systems and Structures 12, pp. 553-565, 2001.

${ }^{8} \mathrm{Su}, \mathrm{Z}$. and Ye, L. Identification of Damage Using Lamb Waves: From Fundamentals to Applications, Springer Verlag, London, UK, 2009.

${ }^{9}$ Chang, Fu-Kuo eds, Structural Health Monitoring: Current Status and Perspectives, CRC Press, April 1998.

${ }^{10}$ Giurgiutiu, V. Structural Health Monitoring with Piezoelectric Wafer Active Sensors; Academic Press: Burlington, MA, USA, 2007.

${ }^{11}$ Davis, M. A. and Kersey, A.D., "All-fiber Bragg grating strain-sensor demodulation technique using a wavelength division coupler," Electronics Letters, 30, pp. 75-77, 1994.

${ }^{12}$ Udd, E., ed., Fiber Optic Sensors, An Introduction for Engineers and Scientists, John Wiley \& Sons Ltd, New York, New York, 1991.

${ }^{13}$ Udd, E., ed., Fiber Optic Smart Structures, John Wiley \& Sons Ltd, New York, New York, 1995.

${ }^{14}$ Al-Chalabi, S., Culshaw, B., and Davies, D., "Partially Coherent Sources in Interferometric Sensors," Proceedings of the 1st International Conference on Optical Fiber Sensors (IEEE Conf. Publ. No. 221), IEEE, London, United Kingdom, pp. 1332-1335, 1983.

${ }^{15}$ Inaudi, D., "State of the art in fiber optic sensing technology and EU structural health monitoring projects." Proceedings of Structural Health Monitoring and Intelligent Infrastructure (SHMII), Tokyo, Japan, pp.191-198, 2003.

${ }^{16}$ Beta, D.C., Thursby, G., Culshaw, B., and Staszewski, W.J., “Acousto-ultrasonic sensing using fiber Bragg gratings.” Smart Materials and Structures, VO1. 12, pp. 122-128, 2003.

${ }^{17}$ Nelson, D.V., Makino, A., Lawrence, C., Seim, J.M., Schulz, W., and Udd, E., "Determination of the K-Matrix for the Multiparameter fiber grating sensor in AD072 fibercore fiber," Proc. SPIE, 3489, 79, 1998.

${ }^{18}$ Udd, E., Schulz, W.L., Seim, J.M., Haugse, E., Trego, A., Johnson, P.E., Bennett, T.E., Nelson, D.V., and Makino, A. "Multidimensional strain field measurements using fiber optic grating sensors," Proc. SPIE, 3986, 254, 2000.

${ }^{19}$ Schulz, W.L., Udd, E., Seim, J.M., Trego, A., and Perez, I.M., "Progress on monitoring of adhesive joints using multi-axis fiber grating densors," Proc. SPIE 3991, 52, 2000.

${ }^{20}$ Udd, E., "Review of multi-parameter fiber grating sensors," Proc. SPIE, 6706, 2007.

${ }^{21}$ Mawatari, T. and Nelson, D., "A multi-parameter Bragg grating fiber optic sensor and triaxial strain measurement," Smart Materials and Structures, Vol. 17, 035033, 2008.

${ }^{22}$ Lin, X and Yuan, F.G., "Diagnostic Lamb waves in an integrated piezoelectric sensor/actuator plate: analytical and experimental studies," Smart Materials and Structures 10: 907-913, 2010.

${ }^{23}$ Giurgiutiu V, Bao J and Zhao W., "Piezoelectric wafer active sensor embedded ultrasonics in beams and plates," Experimental Mechanics 43: 428-449, 2003.

${ }^{24}$ Roh Y-S, Chang F-K, "Effect of impact damage on Lamb wave propagation in laminated composites," In: Sun C (ed.) Proceedings of the ASME International Mechanical Engineering Congress and Exposition (Dynamic Responses and Behavior of Composites), San Francisco, CA, USA, November 12-17, pp. 127-138, 1995. 